\title{
Inadequate preoperative nutrition might be associated with acute kidney injury and greater illness severity postoperatively
}

\author{
Kurt D. Piggott, MD, ${ }^{\mathrm{a}, \mathrm{b}}$ Anne Liu, BS, ${ }^{\mathrm{b}}$ Jessica Monczka, RD, ${ }^{\mathrm{c}}$ Harun Fakioglu, MD, ${ }^{\mathrm{a}, \mathrm{b}}$ \\ Sukumar Suguna Narasimhulu, MD, ${ }^{\mathrm{a}, \mathrm{b}}$ Kamal Pourmoghadam, MD, ${ }^{\mathrm{b}, \mathrm{d}}$ and William DeCampli, MD, PhD ${ }^{\mathrm{b}, \mathrm{d}}$
}

\section{ABSTRACT}

Objective: Nutrition is vital for maintaining optimal cellular and organ function, particularly in neonates who undergo cardiac surgery. Achieving nutritional goals preoperatively can be challenging because of fluid restrictions, suboptimal oral intake, and concerns for inadequate gastrointestinal circulation. We examined preoperative caloric intake and its effects on postoperative course in neonates who underwent cardiac surgery.

Methods: We retrospectively reviewed records of neonates (younger than 30 days) who underwent congenital heart surgery requiring cardiopulmonary bypass from 2008 to 2014 at Arnold Palmer Hospital for Children. Data on multiple nutritional and postoperative variables were collected. Study outcomes included hospital length of stay, duration of mechanical ventilation, and acute kidney injury (AKI).

Results: Records of 95 neonates were reviewed. Sixty-six patients $(69.5 \%)$ with a median age of 5 days did not achieve preoperative caloric goal, whereas 29 patients $(30.5 \%)$ with a median age of 11 days did. Of those who achieved caloric goal, $6(20.6 \%)$ achieved it via total parental nutrition, $9(31.1 \%)$ with a combination of total parental nutrition and enteral feeds, and $14(48.3 \%)$ via enteral route. There was a significant difference in peak lactate $(P=.002)$, inotropic score $(P=.02)$, and duration of mechanical ventilation $(P=.013)$ between those who did and did not achieve caloric goal. In multivariable analysis we found that failure to achieve caloric goal preoperatively was independently associated with stage 2 or 3 AKI $(P=.04$; odds ratio, $4.48 ; 95 \%$ confidence interval, $1.02-$ $19.63)$ and younger age at the time of surgery $(P<.001$; odds ratio, $0.12 ; 95 \%$ confidence interval, 0.04-0.33).

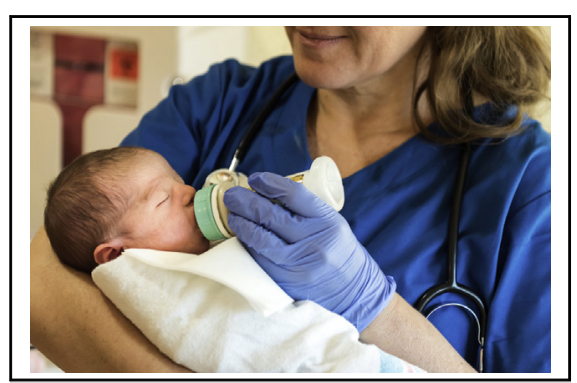

Kurt D. Piggott, MD

\section{Central Message}

In neonates who undergo cardiac surgery, failure to achieve preoperative caloric goal was independently associated with the development of postoperative acute kidney injury.

\section{Perspective}

Neonates who require cardiac surgery often have significant obstacles that limit the ability to achieve preoperative nutritional goals. Although much is known about postoperative nutrition, little is published regarding preoperative caloric goal and its effect on postoperative course.

See Editorial Commentary page 2110.

Conclusions: Failure to achieve preoperative caloric goal might contribute to development of AKI and might be associated with greater severity of illness postoperatively. (J Thorac Cardiovasc Surg 2018;155:2104-9)

Adequate nutrition is vital to the newborn. The critically ill neonate might have increased energy requirements combined with an inability to intake sufficient calories. This

From the ${ }^{\mathrm{a}}$ Pediatric Cardiac Intensive Care, ${ }^{\mathrm{c}}$ Pediatric Cardiac Nutrition, ${ }^{\mathrm{d}}$ Pediatric Cardiothoracic Surgery, Arnold Palmer Hospital for Children, and ${ }^{\mathrm{b}}$ University of Central Florida College of Medicine, Orlando, Fla.

Received for publication March 19, 2017; revisions received Dec 10, 2017; accepted for publication Dec 16, 2017; available ahead of print Feb 1, 2018.

Address for reprints: Kurt D. Piggott, MD, Pediatric Cardiac Intensive Care, Arnold Palmer Hospital for Children, University of Central Florida College of Medicine, Orlando, FL (E-mail: kurtpiggott@yahoo.com).

0022-5223/\$36.00

Copyright (c) 2017 by The American Association for Thoracic Surgery

https://doi.org/10.1016/j.jtcvs.2017.12.080 can lead to a persistent catabolic state, decreased organ function, and poor weight gain. ${ }^{1-3}$ Many neonates born with congenital heart disease suffer from such a scenario. In addition to a severe caloric intake/expenditure imbalance, they often have ductal-dependent circulations leading to tachypnea, inadequate gastrointestinal blood

Scanning this $Q R$ code will take you to a supplemental video for the article. 


\section{Abbreviations and Acronyms \\ AKI = acute kidney injury \\ NEC $=$ necrotizing enterocolitis \\ STAT $=$ Society of Thoracic Surgeons-European \\ Association for Cardio-Thoracic Surgery \\ Congenital Heart Surgery \\ $\mathrm{TPN}=$ total parenteral nutrition}

flow resulting in bowel ischemia, and heart failure. As a result, these patients are often fluid-restricted, further hindering the clinician's ability to deliver adequate calories.

Although these challenges are not unique to a single institution, preoperative feeding practices vary significantly among programs. ${ }^{4,5}$ Some programs choose not to feed neonates orally preoperatively because of concerns for bowel ischemia and the development of necrotizing enterocolitis (NEC). This forces clinicians to deliver calories via total parenteral nutrition (TPN). However, although TPN might decrease the risk of NEC, challenges remain in delivering goal calories because of fluid restrictions, the need for central access, and a limited amount of time to advance calories before cardiac surgery. Additionally, TPN can be expensive and prolonged use can lead to cholestasis and hepatic injury.

The ideal way to measure preoperative nutritional status and degree of catabolic state has yet to be determined. Previous studies have used World Health Organization z-score, using preoperative triceps skin fold measurements in children who underwent congenital heart surgery as a measure of nutritional status. They reported that malnutrition was associated with worse clinical outcomes as well as increased duration of inotropic support and brain natriuretic peptide levels, and decreased myocardial function. ${ }^{7}$ Others have used weight for age z-score preoperatively and reported that poor preoperative nutritional status are associated with adverse short- and long-term outcomes on those who undergo congenital heart surgery. ${ }^{8}$ Many neonates without disease face a catabolic state during the first 2 weeks of postnatal life, particularly breast-fed babies, resulting in weight loss during this time. However, those with critical illness undergo a much more complex stress response that likely sets off an inflammatory cascade as well as uncouples normal energy, protein, and micronutrient metabolism., Although the exact process that occurs is poorly understood, it has yet to be determined if achieving caloric goal before surgery could blunt this catabolic response and perhaps have a positive effect on postoperative course.

The objectives of this study were: (1) To evaluate how successful our program is at achieving preoperative caloric goal in neonates who require surgery for congenital heart disease, and (2) to determine if failing to achieve preoperative caloric goal will have detrimental effects on postoperative course after congenital heart surgery.

\section{METHODS}

We retrospectively reviewed the electronic medical records of neonates admitted to our institution for cardiac surgery who required cardiopulmonary bypass between January 1, 2008 to December 31, 2014. This study was approved by the institutional review board at Arnold Palmer Medical Center and the University of Central Florida College of Medicine in January 2016 Inclusion criteria included all neonates younger than 30 days of age with congenital heart disease requiring cardiac surgery at Arnold Palmer Hospital for Children. Exclusion criteria included any patient who underwent surgery that did not require cardiopulmonary bypass. Preoperative variable data collected included: age at surgery, weight, height, cardiac lesion, prematurity, preoperative mechanical ventilation, preoperative acute kidney injury (AKI), and preoperative lactate. Postoperative variable data collected included: inotropic score, duration of mechanical ventilation, hospital length of stay, Society of Thoracic Surgeons-European Association for CardioThoracic Surgery Congenital Heart Surgery (STAT) mortality categories (STAT 4/5 category), peak lactate, reintubation, cardiopulmonary resuscitation, AKI, mortality, need for cardiopulmonary support (also known as extracorporeal membrane oxygenation), arrhythmia, NEC, catheterization/ reoperation, and sternal wound infection.

Nutritional variable data collected included the following: (1) Achievement of caloric goal in the 48 hours before cardiac surgery determined by the caloric goal outlined by the cardiac intensive care nutrition team. Calorie goals were determined on the basis of using 1.2 times the dietary reference intake, resting energy expenditure, and age. In general that resulted in a goal of 120 kilocalories per kilogram per day for extubated patients and slightly less for intubated and sedated patients because of a lower resting energy expenditure. Also patients who received exclusively TPN would have slightly lower calorie goals because $10 \%$ to $15 \%$ of energy expenditure in neonates is used for enteral digestion/absorption. The exact calorie goal for each patient was obtained from the nutrition note in the electronic medical record. (2) Achievement of protein goal: protein goal was $3 \mathrm{~g} / \mathrm{kg} / \mathrm{d}$. The patient was considered to have achieved calorie and protein goal if they reached the recommended goal within 48 hours before surgery. AKI was defined using the Kidney Disease: Improving Global Outcomes criteria, which uses change in creatinine from baseline and measures of urine output to categorize patients into stage 1, 2, or 3 AKI. Only those with stage 2 or 3 AKI were analyzed because these stages reflect severe AKI.

\section{Statistical Analysis}

SPSS version 22.0 (IBM Corp, Armonk, NY) was used for statistical analysis. Categorical data are presented as frequencies and percentages, and between group comparisons were made using $\chi^{2}$ or Fisher exact tests, as appropriate. Continuous data are reported as median value with interquartile range (25th-75th percentile). Variables were tested to determine if they were normally distributed. Those that were not underwent statistical analysis with nonparametric test for independent samples. All $P$ values $<.05$ were considered statistically significant. Variables with a $P$ value of .1 or less after univariate analysis underwent multivariable logistic regression analysis.

\section{Multivariable Analysis}

For the multivariable analysis, we used binary logistic regression analysis. For the dependent variables, stage 2 as well as $3 \mathrm{AKI}$ and preoperative calorie goal achieved were handled as binary (yes/no) variables. For the analysis using preoperative calorie goal as the dependent variable, the covariates and how they were defined included cardiac arrest (nominal), STAT category $4 / 5$ (nominal), stage 2 or 3 AKI (nominal), inotropic score (scale), age at surgery $\geq 7$ days (nominal), and sternal wound infection 
TABLE 1. Patient characteristics

\begin{tabular}{lccc}
\hline Variable & $\begin{array}{c}\text { Preoperative } \\
\text { calorie goal } \\
\text { achieved }(\mathbf{n}=\mathbf{2 9})\end{array}$ & $\begin{array}{c}\text { Preoperative } \\
\text { calorie goal not } \\
\text { achieved }(\mathbf{n}=\mathbf{6 6})\end{array}$ & $\boldsymbol{P}$ value \\
\hline Age, d & $11(8-21)$ & $5(3-7)$ & $<.001$ \\
Weight, $\mathrm{kg}$ & $2.9(2.7-3.2)$ & $3.2(2.96-3.6)$ & .006 \\
Height, $\mathrm{cm}$ & $49(48-51)$ & $49.5(47.6-51)$ & .64 \\
Male sex & $12(41.3)$ & $38(57.5)$ & .28 \\
\hline
\end{tabular}

Data presented as value with percentage or median with interquartile range.

(nominal). In the analysis using stage 2 or $3 \mathrm{AKI}$ as the dependent variable, the covariates and how they were defined included selective cerebral perfusion (nominal), cardiac arrest (nominal), preoperative calorie goal (nominal), cardiopulmonary bypass time (scale), and age in days at surgery (scale). There were no missing data in either analysis.

\section{RESULTS}

We included 95 neonates in the study. Twenty-nine patients $(30.5 \%)$ successfully achieved preoperative caloric goal, whereas $66(69.5 \%)$ did not. Table 1 shows baseline characteristics between those who achieved caloric goal and those who did not. In terms of baseline characteristics, there was a statistically significant difference in patient age between those who did and did not achieve caloric goal (median 11 vs 5 days, respectively; $P<.001)$ and weight $(2.9 \mathrm{~kg}$ vs $3.2 \mathrm{~kg} ; P=.006)$. No patient from either group experienced AKI preoperatively.
Univariate analysis showed a statistically significant difference between the group who achieved caloric goal and the group that did not in terms of postoperative inotrope score $(P=.02)$ and peak lactate $(P=.002$; Table 2$)$. Postoperative hospital length of stay (median 43 vs 26 days; $P=.09$ ), whereas longer in the group who did not achieve caloric goal, was not statistically significant. Duration of mechanical ventilation (median 7 vs $4 ; P=.013$ ) was longer in patients who did not achieve their caloric goal preoperatively and reached statistical significance. There were also an increased number of deaths (5 vs $1 ; P=.33$ ) in the group that did not achieve preoperative caloric goal, although not statistically significant (Table 2).

Sixty-six patients $(69.5 \%)$ achieved their preoperative protein goal, whereas $29(30.5 \%)$ did not. Univariate analysis of the aforementioned postoperative variables showed no significant difference between the group that met the protein goal preoperatively and the group that did not.

Of those achieving caloric goal, $6(20.6 \%)$ achieved it via TPN, $9(31.1 \%)$ via a combination of TPN and enteral feeds, whereas $14(48.3 \%)$ achieved caloric goal via enteral feeding.

Multivariable logistic regression analysis with preoperative calorie goal as the dependent variable revealed an independent association with age $\geq 7$ days at the time of surgery $(P<.001$; odds ratio, $0.12 ; 95 \%$ confidence interval, $0.04-$ 0.33 ; Table 3 ). The variables included in the analysis

TABLE 2. Preoperative and postoperative variables and ICU outcomes

\begin{tabular}{|c|c|c|c|}
\hline Variable & Preoperative calorie goal achieved $(n=29)$ & Preoperative calorie goal not achieved $(n=66)$ & $P$ value \\
\hline \multicolumn{4}{|l|}{ Preoperative } \\
\hline Age at surgery $\geq 7 \mathrm{~d}$ & $22(75.8)$ & $18(27.3)$ & $<.001$ \\
\hline Ventilation (yes) & $6(20.7)$ & $15(22.7)$ & .52 \\
\hline Prematurity & $6(20.7)$ & $5(7.6)$ & .09 \\
\hline AKI (KDIGO) & 0 & 0 & N/A \\
\hline Peak lactate 48-hour PTS & $1(0.5-2)$ & $1.5(1-3)$ & .12 \\
\hline \multicolumn{4}{|l|}{ Postoperative } \\
\hline ECMO & $1(3.4)$ & $5(7.6)$ & .66 \\
\hline Single ventricle & $12(29)$ & $34(43)$ & .17 \\
\hline Cardiac arrest & $3(10.3)$ & $8(12.1)$ & .80 \\
\hline Arrhythmia & $8(19)$ & $23(29.1)$ & .63 \\
\hline Cath/reoperation & $3(10.3)$ & $18(27.2)$ & .11 \\
\hline Reintubation & $5(17.2)$ & $14(21.2)$ & .78 \\
\hline SWI & $4(13.7)$ & $5(7.6)$ & .45 \\
\hline Stage $2 / 3$ AKI (KDIGO) & $6(20.7)$ & $28(42.4)$ & .06 \\
\hline STAT $4 / 5$ & $16(55.2)$ & $47(71.2)$ & .1 \\
\hline Inotropic score & $10(7-13)$ & $15(10-21)$ & .02 \\
\hline Peak lactate & $3.5(2.6-5)$ & $4.95(3.7-6)$ & .002 \\
\hline \multicolumn{4}{|l|}{ ICU outcomes } \\
\hline HLOS & $26(20-47)$ & $43(22-61)$ & .09 \\
\hline DMV & $4(3-7)$ & $7(4-17)$ & .013 \\
\hline Mortality & $1(3.4)$ & $5(7.6)$ & .66 \\
\hline
\end{tabular}

Data are presented as value with percentage or median with interquartile range. AKI, Acute kidney injury; $K D I G O$, Kidney Disease: Improving Global Outcomes; $N / A$, not applicable; PTS, before surgery; ECMO, extracorporeal membrane oxygenation; Cath, cardiac catheterization; SWI, sternal wound infection; STAT, Society of Thoracic Surgeons-European Association for Cardio-Thoracic Surgery Congenital Heart Surgery; ICU, intensive care unit; HLOS, hospital length of stay; DMV, duration of mechanical ventilation. 
TABLE 3. Multivariable analysis with failure to achieve preoperative calorie goal as dependent variable

\begin{tabular}{lcc}
\hline \multicolumn{1}{c}{ Variable } & Adjusted OR $(\mathbf{9 5} \%$ CI) & $\boldsymbol{P}$ value \\
\hline STAT 4/5 & $2.3(0.81-6.5)$ & .12 \\
Age at surgery $\geq 7 \mathrm{~d}$ & $0.12(0.04-0.33)$ & $<.001$ \\
\hline $\begin{array}{l}\text { OR, Odds ratio; } C I \text {, confidence interval; STAT, Society of Thoracic Surgeons-Euro- } \\
\text { pean Association for Cardio-Thoracic Surgery Congenital Heart Surgery. }\end{array}$
\end{tabular}

included age $\geq 7$ days at the time of surgery, STAT $4 / 5$ category, single ventricle anatomy, and preoperative mechanical ventilation.

Multivariable logistic regression analysis using stage 2 or $3 \mathrm{AKI}$ as the dependent variable showed failure to achieve preoperative caloric goal to be independently associated with stage 2 or 3 AKI $(P=.04$; odds ratio, $4.48 ; 95 \%$ confidence interval, $1.02-19.63)$. We performed the analysis using variables that have been previously reported in other studies to be associated with AKI. These variables included cardiopulmonary bypass, selective cerebral perfusion, cardiac arrest, inotropic score, and STAT category (Table 4).

In an attempt to better understand if preoperative condition or complexity of cardiac lesion contributed to postoperative AKI, STAT category was analyzed. The median STAT category in both groups was $4(P=.07)$, without a statistically significant difference. Further data analysis of AKI and preoperative calorie goal among the individual STAT categories (Table 5) showed a statistically significant difference between those who did and did not achieve caloric goal in STAT 4 and STAT 5 patients. Additionally, a subanalysis of patient age at the time of cardiac surgery was performed individually for STAT categories 3,4 , and 5 to determine if there was a significant difference between those who achieved preoperative calorie goal and those who did not. There was a significant difference in age between the groups for all 3 STAT categories (Table 6).

\section{DISCUSSION}

Our study highlights the challenges that clinicians face when trying to achieve caloric goal preoperatively. In our cohort, 29 of 95 neonates $(30.5 \%)$ achieved caloric goal before undergoing cardiac surgery. This is a reflection of the obstacles faced such as fluid restrictions, heart failure, limited ability to tolerate enteral feeds, tachypnea,

TABLE 4. Multivariable analysis with stage $2 / 3 \mathrm{AKI}$ as the dependent variable

\begin{tabular}{lcc}
\hline \multicolumn{1}{c}{ Variable } & Adjusted OR $(\mathbf{9 5} \% \mathbf{C I})$ & $\boldsymbol{P}$ value \\
\hline $\begin{array}{l}\text { Preoperative calorie goal not } \\
\text { met }\end{array}$ & $4.48(1.02-19.63)$ & .04 \\
Inotropic Score & $2.01(1.19-3.43)$ & .01 \\
\hline$O R$, Odds ratio; $C I$, confidence interval. &
\end{tabular}

TABLE 5. Analysis of stage 2 or 3 AKI/preoperative calorie goal according to STAT category

\begin{tabular}{|c|c|c|c|c|c|}
\hline & $+\mathbf{A K I} /+\mathbf{c a l}$ & $+\mathbf{A K I} /-\mathbf{c a l}$ & $-\mathbf{A K I} /+\mathbf{c a l}$ & $-\mathbf{A K I} /-$ cal & $\begin{array}{c}P \\
\text { value }\end{array}$ \\
\hline STAT 2 & 3 & 0 & 2 & 5 & .17 \\
\hline STAT 3 & 1 & 3 & 7 & 11 & .53 \\
\hline STAT 4 & 1 & 10 & 8 & 11 & .06 \\
\hline STAT 5 & 1 & 15 & 6 & 11 & .08 \\
\hline ALL & 6 & 28 & 23 & 38 & .06 \\
\hline
\end{tabular}

$\chi^{2}$ Test was used. + Signifies yes patients developed AKI and/or yes achieved preoperative caloric goal. - Signifies no patients did not develop AKI and/or no did not achieve preoperative caloric goal. AKI, Acute kidney injury; cal, preoperative calorie goal; STAT, Society of Thoracic Surgeons-European Association for Cardio-Thoracic Surgery Congenital Heart Surgery.

unbalanced circulation causing limited gastrointestinal perfusion, and increased energy expenditure. Additionally, because of limited tolerance of enteral feeds and poor oral intake, calories are often delivered via TPN. Challenges reaching caloric goal via TPN can result from lack of central access, which is essential for the advancement of calories as well as insufficient time preoperatively to allow advancement of calories to goal. In our cohort, a total of 66 patients $(69.5 \%)$ achieved their preoperative protein goal, whereas $29(30.5 \%)$ did not. This finding highlights the fact that even with significant fluid restrictions that are often present many times protein goal can still be achieved.

Although much focus has been placed on postoperative nutrition, little has been written regarding preoperative caloric intake. Because of the physiologic state of the neonate requiring surgery for congenital heart disease and the short duration allowed to deliver calories, it is unlikely that the clinician will be able to reverse any catabolic state present preoperatively before surgery. As previously mentioned, most neonates, including those without critical illness, undergo a period of catabolism during the first couple of weeks of postnatal life. This is exacerbated by critical illness and because achieving caloric goal postoperatively often takes time, it is vitally important to optimize nutritional status before surgery.

In 2014, Radman et al used World Health Organization zscore and measured preoperative triceps skin folds in children who underwent surgery for congenital heart surgery and reported malnutrition to be associated with worse clinical outcomes. ${ }^{7}$ In 2015 Mitting et al used weight-for-age z

TABLE 6. Age (days) at surgery according to STAT category

\begin{tabular}{lccc}
\hline & Age at surgery/+cal & Age at surgery/-cal & $\boldsymbol{P}$ value \\
\hline STAT 3 & $9(6-15), \mathrm{n}=8$ & $4(3-6), \mathrm{n}=14$ & .008 \\
STAT 4 & $9(8-15), \mathrm{n}=9$ & $5(4-6), \mathrm{n}=26$ & .006 \\
STAT 5 & $9(6-11), \mathrm{n}=7$ & $6(4.5-7), \mathrm{n}=26$ & .014 \\
\hline + Signifies yes patients developed AKI and/or yes achieved preoperative caloric goal. \\
- Signifies no patients did not develop AKI and/or no did not achieve preoperative \\
caloric goal. cal, Preoperative calorie goal; STAT, Society of Thoracic Surgeons- \\
European Association for Cardio-Thoracic Surgery Congenital Heart Surgery.
\end{tabular}


score preoperatively and reported that poor preoperative nutritional status has adverse short and long-term outcomes on those undergoing congenital heart surgery. ${ }^{8}$ To our knowledge, our study is the first to evaluate the separate effects of achieving preoperative calorie and protein goal on postoperative course.

The most interesting finding in our study is the fact that failure to achieve preoperative caloric goal was independently associated with the development of AKI in multivariable analysis. To our knowledge this has never been reported. Statistically significant differences in inotropic score and peak lactate were noted between the groups. These are signs of increased severity of illness postoperatively. After removal of the non-cardiopulmonary bypass patients, there was not a statistically significant difference in the number of STAT $4 / 5$ category patients between the groups. However, we still believe that some, if not all of these findings could be explained by the greater complexity of congenital heart disease and more complex surgery. This combined with the statistically significant difference in age noted in each of the individual STAT categories could indicate a sicker preoperative patient requiring earlier surgery and resulting in an increased likelihood of AKI and a greater illness severity postoperatively. Another interesting finding is the statistically significant difference in preoperative weight between the groups. A possible explanation for this finding is the greater percentage of premature neonates in the group that achieved the preoperative calorie goal. Although prematurity often results in delaying surgery and could ultimately have an effect on the increased age at surgery, we performed an analysis with the premature neonates excluded and there remained a significant difference in age between the 2 groups.

Clinical and experimental models of protein-calorie malnutrition have suggested significant alterations in renal hemodynamics and renal concentrating ability. Children and adults with malnutrition have been shown to decrease glomerular filtration rate and experimental models have suggested changes in the renin-angiotensin system, renal prostaglandins, and overall changes in renal function. ${ }^{11-13}$ Although the exact mechanism might remain poorly understood, this finding is nonetheless very important. Neonates who undergoing surgery for congenital heart disease remain one of the most vulnerable subsets of patients for the development of AKI, with an incidence reported between $15 \%$ and $59 \%$. Additionally, the development of AKI is associated with fluid overload and significant morbidity and mortality (Video 1 ). ${ }^{14-20}$

\section{CONCLUSIONS}

This retrospective study highlights an important yet understudied obstacle that affects neonates who undergo surgery for congenital heart disease. Although our data

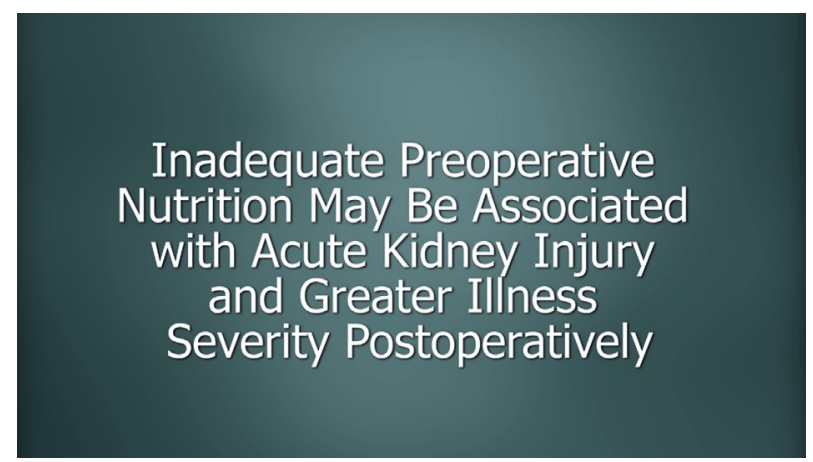

VIDEO 1. The video explains challenges encountered achieving preoperative caloric goal in neonates who undergo surgery for congenital heart disease and the consequences of failing to do so such as the risk of developing stage 2/3 acute kidney injury and increased illness severity postoperatively. Video available at: http://www.jtcvsonline.org/article/ S0022-5223(17)33058-1/fulltext.

are interesting, it is merely preliminary data and is insufficient to recommend clinical change. Our study underscores the difficulties clinicians face in achieving adequate nutrition preoperatively and the potential clinical effects it can have on these fragile patients. We hope that this early investigative line of thought will lead to further prospective, multi-institutional studies. We hope that this study can function as a starting point for further investigation, perhaps leading to identification of lab values that could alert clinicians to the presence of a severe catabolic state or even the ability to predict or identify those at risk for postoperative $\mathrm{AKI}$ and increased illness severity.

\section{Limitation}

There are significant limitations to our study, including the retrospective nature and the fact that it is from a single institution. Additionally, we do not have a feeding protocol that we follow preoperatively so decisions on mode of calorie delivery and speed of advancement are left up to the attending physician on service, which make the results susceptible to bias. Additionally, the fact that there was a statistically significant difference in age between the groups that did and did not achieve preoperative calorie goal could be a potential source of bias.

\section{Conflict of Interest Statement}

Authors have nothing to disclose with regard to commercial support.

\section{References}

1. Cameron JW, Rosenthal A, Olson AD. Malnutrition in hospitalized children with congenital heart disease. Arch Pediatr Adolesc Med. 1995;149:1098-102.

2. Mehta NM, Duggan CP. Nutritional deficiencies during critical illness. Pediatr Clin North Am. 2009;56:1143-60.

3. Singh N, Gupta D, Aggarwal AN, Agarwal R, Jindal SK. An assessment of nutritional support to critically ill patients and its correlation with outcomes in a respiratory intensive care unit. Respir Care. 2009;54:1688-96. 
4. Alten JA, Rhodes LA, Tabbutt S, Cooper DS, Graham EM, Ghanayem N, et al. Perioperative feeding management of neonates with CHD: analysis of the Pediatric Cardiac Critical Care Consortium (PC4) registry. Cardiol Young. 2015;25: 1593-601.

5. Lambert LM, Pike NA, Medoff-Cooper B, Zak V, Pemberton VL, YoungBorkowski L, et al. Variation in feeding practices following the Norwood procedure. J Pediatr. 2014;164:237-42.e1.

6. Naini BV, Lassman CR. Total parenteral nutrition therapy and liver injury: a histopathologic study with clinical correlation. Hum Pathol. 2012;43:826-33.

7. Radman M, Mack R, Barnoya J, Castañeda A, Rosales M, Azakie A, et al. The effect of pre-operative nutritional status on post-operative outcomes in childrens undergoing surgery for congenital heart defects in San Francisco and Guatemala City. J Thorac Cardiovasc Surg. 2014;147:442-50.

8. Mitting R, Marino L, Macrae D, Shastri N, Meyer R, Pathan N. Nutritional status and clinical outcome in postterm neonates undergoing surgery for congenital heart disease. Pediatr Crit Care Med. 2015;16:448-52.

9. Ramel SE, Brown LD, Georgieff MK. The impact of neonatal illness on nutritional requirements-one size does not fit all. Curr Pediatr Rep. 2014;2:248-54.

10. Marchini G, Persson B, Uvnas-Moberg K. Plasma gastrin and somatostatin levels in infants during the first four postnatal days. J Dev Physiol. 1990;14:157-62.

11. Benabe JE, Martinez-Maldonado M. The impact of malnutrition on kidney function. Miner Electrolyte Metab. 1998;24:20-6.

12. Nascimento Gomes G, Sato H, Aihara AY, Cavanal MF, Zaladek Gil F. Malnutrition as an additional risk factor to gentamicin nephrotoxicity. Int Braz J Urol. 2002;28:265-70.

13. Toigo G, Aparicio M, Attman PO, Cano N, Cianciaruso B, Engel B, et al. Expert Working Group report on nutrition in adult patients with renal insufficiency. Clin Nutr. 2000;19:197-207.
14. Aydin S, Seiden H, Blaufox A, Parnell VA, Choudhury T, Punnoose A, et al Acute kidney injury after surgery for congenital heart disease. Ann Thorac Surg. 2012;94:1589-95.

15. Andreoli SP. Acute kidney injury in children. Pediatr Nephrol. 2009;24: 253-63.

16. Blinder JJ, Goldstein SL, Lee VV, Baycroft A, Fraser CD, Nelson D, et al Congenital heart surgery in infants: effects of acute kidney injury on outcomes. J Thorac Cardiovasc Surg. 2012;143:368-74.

17. Zappitelli M, Bernier PL, Saczkowski RS, Tchervenkov CI, Gottesman R, Dancea A, et al. A small post-operative rise in serum creatinine predicts acute kidney injury in children undergoing cardiac surgery. Kidney Int. 2009;76: 885-92.

18. Akcan-Arikan A, Zappitelli M, Loftis LL, Washburn KK, Jefferson LS, Goldstein SL. Modified RIFLE criteria in critically ill children with acute kidney injury. Kidney Int. 2007;71:1028-35.

19. Li S, Krawczeski CD, Zappitelli M, Devarajan P, Thiessen-Philbrook H Coca SG, et al. Incidence, risk factors, and outcomes of acute kidney injury after pediatric cardiac surgery: a prospective multicenter study. Crit Care Med. 2011 39:1493-9.

20. Piggott KD, Soni M, DeCampli WD, Ramirez JA, Holbein D, Fakioglu H, et al. Acute kidney injury and fluid overload in neonates following surgery for congenital heart disease. World J Pediatr Congenit Heart Surg. 2015;6: 401-6.

Key Words: neonate, preoperative malnutrion, acute kidney injury, congenital heart disease, total parenteral nutrition 